\title{
Replication of Respiratory Syncytial Virus Is Inhibited by the Host Defense Molecule Viperin
}

\author{
Glen McGillivary ${ }^{a} \quad$ Zachary B. Jordan ${ }^{a} \quad$ Mark E. Peeples ${ }^{b, c} \quad$ Lauren O. Bakaletz ${ }^{a, c}$ \\ Centers for ${ }^{\mathrm{a}}$ Microbial Pathogenesis and ${ }^{b}$ Vaccines and Immunity, The Research Institute, Nationwide Children's \\ Hospital, and 'Department of Pediatrics, The Ohio State University College of Medicine, Columbus, Ohio, USA
}

\section{Key Words}

Antiviral proteins $\cdot$ Bronchiolitis $\cdot$ Chinchilla $\cdot$ Otitis media . Pneumonia $\cdot$ Respiratory syncytial virus $\cdot$ Respiratory tract $•$ Viperin

\begin{abstract}
Respiratory syncytial virus (RSV) is an important viral pathogen of otitis media, bronchiolitis, and pneumonia. As infection of the upper airways is a precondition for the development of these diseases, understanding RSV pathogenesis and the host response induced by RSV in this niche may enable the development of novel therapeutic strategies against this virus. We have used a microarray approach and showed that expression of the gene that encodes the antiviral protein viperin was significantly upregulated in the chinchilla nasopharynx up to 1 week after RSV challenge. Overexpression of human viperin in vitro diminished the ability of RSV to infect HeLa or A549 cells. Furthermore, transduction of the chinchilla airways with a recombinant adeno-associated virus vector that encodes viperin resulted in reduced titers of RSV in the nasopharyngeal lavage fluid. Collectively, these data indicated that viperin plays a significant role in the innate immune defense against RSV.
\end{abstract}

Copyright $\odot 2012$ S. Karger AG, Basel

\section{KARGER}

Fax +41613061234

E-Mail karger@karger.ch

www.karger.com
(C) 2012 S. Karger AG, Basel

1662-811X/13/0051-0060\$38.00/0

Accessible online at:

www.karger.com/jin

\section{Introduction}

Respiratory syncytial virus (RSV) is a nonsegmented, negative-strand RNA virus that belongs to the Pneumovirinae subfamily of the Paramyxoviridae family. This virus is a major cause of respiratory infections in infants and the elderly, and bronchiolitis and pneumonia caused by RSV are the most frequent reasons for hospitalization of young children [1]. RSV infects more than $70 \%$ of children in the 1 st year of life and essentially $100 \%$ by the age of 2 . It has been estimated that 34 million new cases of RSV-associated acute lower respiratory tract infections occur worldwide annually in children younger than 5 years, causing 66,000-199,000 deaths [2]. Compounding the significant morbidity and mortality due to RSV is the fact that repeated exposure is common in all age groups, and previous infection does not prevent subsequent infection [3].

RSV is transmitted from person to person via inhalation of respiratory droplets. Initial attachment of the virion involves the viral attachment $G$ glycoprotein, which has been shown to adhere to glycosaminoglycans, particularly heparan sulfate, on the surface of immortalized cells $[4,5]$. This initial interaction enables engagement of the viral fusion F protein, perhaps through the recently 
identified cellular receptor for RSV, human nucleolin [6]. Upon triggering, the viral fusion $\mathrm{F}$ protein refolds resulting in fusion of the virus envelope and the host cell membrane. It is not clear what causes the viral $\mathrm{F}$ protein to trigger, but we have suggested that reduced ionic strength or molarity of the microenvironment, such as presence within an endosome, may be involved [7]. This fusion event results in the release of the RSV ribonucleoprotein complex into the cytoplasm where mRNA transcription and virus replication are initiated. Virus glycoproteins and ribonucleoprotein complexes are targeted to lipid raft domains at the cell surface, and mature virions subsequently bud and release to infect other cells of the airways [8].

RSV disease pathogenesis is a multifactorial and complex process that includes the ability of the virus to target and replicate in ciliated cells lining the airways and host mobilization of key effectors of the innate and adaptive immune system that mediate viral clearance [9-12]. Host recognition of RSV is essential for regulating the outcome of infection and mediated primarily through toll-like receptor 3 (TLR3) recognition of dsRNA [13] and TLR4 detection of the RSV F protein. Additional TLRs also contribute to the regulation of immune responses to RSV [14, 15]. Signaling through these TLRs initiates a signaling cascade leading to the induction of several transcription factors such as interferon (IFN) regulatory proteins (IRF3 and IFR7), STAT (signal transducer and activator of transcription), JNK (c-Jun $\mathrm{NH}_{2}$-terminal kinase), nuclear factor- $\mathrm{\kappa} \mathrm{B}$, and others that augment expression of proinflammatory genes that include IFN- $\gamma$. In addition, cytoplasmic molecules such as retinoic acid-inducible gene (RIG-I) can become activated in response to the presence of viral nucleic acids within the cell and induce, although weakly, expression of IFN- $\alpha$ and IFN- $\beta$ [16]. IFN production contributes to the RSV-induced altered expression of hundreds of host genes [17, 18], but how this intricate transcriptional response to virus results in an effective antiviral state in the respiratory tract is not currently known.

Recently, the IFN-inducible protein viperin [19-21] was shown to inhibit replication of several viruses that include influenza virus [22], human cytomegalovirus (CMV) [19], hepatitis C virus [23], dengue virus [24], alphaviruses [25], Sindbis virus [26], and human immunodeficiency virus [27]. The exact mechanism whereby viperin inhibits replication for each of these viruses is not known, but it is thought that this protein affects the later stages of the viral life cycle by preventing release of virions from the cell surface. Wang et al. [22] have shown that viperin localizes to lipid droplets within epithelial cells and can bind to and inhibit farnesyl diphosphate synthase (FPPS). FPPS is an enzyme involved in cholesterol and isoprenoid synthesis, and the interaction between viperin and FPPS results in disruption of the organization of lipid raft domains at the cellular phospholipid bilayer [22]. Viperin expression is induced in response to influenza virus infection $[19,23,26,28]$ which facilitates interactions with FPPS and disrupts viral assembly at lipid raft domains [22], a key step in the viral replication cycle.

As relatively little is known about the host response to RSV in the upper airways, we previously characterized the impact that RSV had on the expression of targeted antiviral host defense proteins in this niche $[29,30]$. Here, we increased the breadth of our initial approach and utilized microarray analysis to identify additional effectors of innate immunity whose expression was modulated in response to RSV and who likely exhibited antiviral activity. We present evidence that intranasal challenge of chinchillas with RSV resulted in significant upregulation of $v i$ perin expression in nasopharyngeal mucosa. We show that viperin was produced in every upper airway tissue permissive to infection with RSV [31,32] and that augmented production of viperin in epithelial cells in vitro inhibited the ability of RSV to replicate. Furthermore, recombinant adeno-associated virus (rAAV) vector-mediated overexpression of viperin in chinchilla airways resulted in significantly reduced titers of RSV in nasopharyngeal lavage (NPL) fluids compared to controls. Collectively, our data demonstrate that expression of viperin in the upper airways results in decreased replication of RSV and suggests that viperin plays an important role in host defense against this clinically important pathogen.

\section{Materials and Methods}

Determination of RSV-Mediated Alteration in Viperin Protein Expression

Animal care and all procedures were performed in concordance with institutional and federal guidelines, and were conducted under an approved protocol. To confirm our initial microarray approach (online Supplementary Materials and Methods; see www.karger.com/doi/10.1159/000342473 for all online suppl. material), 2 juvenile chinchillas were inoculated intranasally with 1 $\times 10^{8}$ pfu RSV or viral suspension buffer. Four days after viral challenge, animals were sacrificed and nasopharyngeal mucosae was obtained and stored at $-80^{\circ} \mathrm{C}$. Mucosae were homogenized (Omni International, Kennesaw, Ga., USA) in $200 \mu$ l sterile saline that contained a $1 \times$ protease inhibitor cocktail (Roche Applied Science, Indianapolis, Ind., USA), centrifuged at 3,000 $g$ to pellet cellular debris and the supernatant was retained.

Eighty micrograms of protein from nasopharyngeal mucosa were separated in a 4-20\% mini-protean TGX gel (Bio-Rad, Her- 
cules, Calif., USA). Proteins were transferred to nitrocellulose for $1 \mathrm{~h}$ at $4^{\circ} \mathrm{C}$ at $100 \mathrm{~V}$, and blots were incubated with $3 \%$ skim milk (Fisher Scientific, Pittsburg, Pa., USA) in TTBS (7.88 g Tris- $\mathrm{HCl}$, $25 \mathrm{~g} \mathrm{NaCl}$ and $0.5 \mathrm{ml}$ Tween 20 per liter; Fisher Scientific; pH 7.2) overnight to block non-specific binding of antibody. Blots were incubated with a 1:1,000 dilution of a monoclonal anti-mouse viperin antibody (kind gift from Peter Cresswell) and antibody bound to the membrane was visualized with a 1:10,000 dilution of HRP-conjugated goat anti-mouse IgG (Invitrogen, Carlsbad, Calif., USA) followed by chemiluminescent detection (GE Healthcare, Piscataway, N.J., USA). After capture of signal on film, blots were incubated for $5 \mathrm{~min}$ in $0.2 \mathrm{M} \mathrm{NaOH}$ to strip bound antibodies from the blot. Nitrocellulose was incubated in water for $5 \mathrm{~min}$ and then with $3 \%$ skim milk blocking buffer. To confirm that equivalent protein amounts were loaded on the gel and to provide a standard for normalization of protein expression as assessed via densitometry, blots were incubated with a 1:500 dilution of goat anti-mouse glyceraldehyde-3-phopsphate dehydrogenase (GAPDH; Invitrogen), and immunocomplexes were detected as with viperin. Densitometric analyses of the intensity of protein bands were reported as the ratio of viperin protein abundance from RSV-infected to uninfected cultures normalized against the GAPDH protein.

\section{Relative Expression of Viperin in Upper Airway Tissues}

To determine sites of viperin expression in the respiratory tract, mucosal samples from the nasal septum, ethmoid turbinates, nasoturbinates, nasopharynx, eustachian tube, and lung were dissected from a naive juvenile chinchilla or a chinchilla challenged 4 days prior with $1 \times 10^{8}$ pfu RSV A2. Samples were homogenized and a Western blot was performed to detect chinchilla viperin as described in the previous section. GAPDH was also detected to confirm that equivalent protein amounts were loaded on the gel and to provide a target for normalization of protein expression as assessed via densitometry.

To determine what cell types expressed viperin in the upper airways, we cultured differentiated, pseudostratified, ciliated nasopharyngeal epithelial cells. The conditions required to grow these primary cells and the methods utilized to obtain sections for staining with hematoxylin and eosin are reported in detail elsewhere [33].

\section{Ability of Viperin to Exhibit Anti-RSV Activity in vitro}

A previous report demonstrated that HeLa cells do not express detectable amounts of viperin as assessed by Western blot [19]. As HeLa cells are also permissible to infection with RSV, we utilized this cell culture system to determine if previous overexpression of viperin resulted in a subsequent increase or decrease in the number of RSV-infected cells. We therefore seeded a 96-well plate with $2 \times 10^{4} \mathrm{HeLa}$ cells (ATCC) per well grown in DMEM cell culture medium (Mediatech, Manassas, Va., USA) supplemented with 7.5\% fetal bovine serum (Fisher Scientific) and $100 \mathrm{U}$ penicillin/ streptomycin (Mediatech) per liter and cultivated the cells overnight at $37^{\circ} \mathrm{C}$ with $5 \% \mathrm{CO}_{2}$. The next day, we incubated $100 \mathrm{ng}$ of pGM-17, the viperin overexpression construct, or pcDNA3.1/ nV5-DEST, vector control, with $0.4 \mu \mathrm{l}$ of attractene transfection reagent (Qiagen, Valencia, Calif., USA) in serum- and antibioticfree DMEM medium for $15 \mathrm{~min}$ at $25^{\circ} \mathrm{C}$. Samples that contained pGM-17, pcDNA3.1/nV5-DEST or cell culture medium alone were added to cells overnight to allow for transfection and the cells were then washed 3 times with $1 \times$ DPBS (Mediatech). Cells were inoculated with complete cell culture medium with or without recombinant green fluorescent protein (GFP)-expressing RSV $(\mathrm{MOI}=1)$ for $48 \mathrm{~h}$ [5]. Cells were washed thrice with $1 \times$ DPBS and then $200 \mu \mathrm{l}$ of $0.25 \%$ trypsin-21 mM EDTA in HBSS (Mediatech) were added to detach the cells from the microtiter plate. $\mathrm{HeLa}$ cells were centrifuged at $400 \mathrm{~g}$ for $5 \mathrm{~min}$ at $20^{\circ} \mathrm{C}$ to pellet cells and then washed with $500 \mu \mathrm{l}$ of HBSS that contained $1 \%$ bovine serum albumin (Fisher). This process was repeated two more times and the cells were immediately analyzed by flow cytometry with a BD FACSCalibur. A total of 10,000 viable events were collected for independent assays, and the data were analyzed with FloJo software (TreeStar, Inc., Ashland, Oreg., USA). To assess the relative ability of viperin to inhibit replication of RSV in a respiratory epithelial cell line, we used A549 cells purchased from ATCC, cultivated these cells in DMEM supplemented with $10 \%$ fetal bovine serum, and used the same experimental approach as was used with the HeLa cells.

\section{Construction of a rAAV9 Vector That Overexpressed Viperin}

To initiate studies on the ability of viperin to exhibit anti-RSV activity in vivo, we developed a system to overexpress this protein in the chinchilla airways. In preliminary studies, we demonstrated that challenge of chinchillas with a self-complementary rAAV vector serotype 9 that contained a $g f p$ transgene effectively transduced the chinchilla upper airways. As such, we cloned the human viperin cDNA (clone ID IOH55127) from a Gateway entry vector into an rAAV9 vector backbone. We used Phusion DNA polymerase (New England Biolabs, Ipswich, Mass., USA), the Gateway plasmid, with viperin AAV9 forward (5'-TAATAAACCGGTATGTGGGTGGTTAC-3') and viperin AAV9 reverse primers (5'-TAATAAGCGGCCGCCTACCAATCCAG-3') in a PCR reaction to amplify the viperin open reading frame. Forward and reverse primers were designed to contain an AgeI or NotI restriction site, respectively, and conditions for amplification were an initial denaturation at $98^{\circ} \mathrm{C}$ followed by a 35 -cycle 3-step procedure that consisted of denaturation at $98^{\circ} \mathrm{C}$ for $10 \mathrm{~s}$ followed by annealing at $65^{\circ} \mathrm{C}$ for $30 \mathrm{~s}$ and extension at $72^{\circ} \mathrm{C}$ for $30 \mathrm{~s}$. PCR products were separated in a gel red (Phenix Research Products, Candler, N.C., USA)-stained agarose gel and the Wizard SV gel and PCR clean-up system (Promega, Madison, Wisc., USA) was used to extract and purify amplicons. The plasmid rAAV9.CMV.gfp [Viral Vector Core, The Research Institute at Nationwide Children's Hospital (TRINCH)] and amplified DNA fragments were digested with AgeI and NotI, gel extracted again, and ligated (New England Biolabs). Plasmids obtained from this cloning step were transformed into Escherichia coli Top 10 cells for growth on LB supplemented with $50 \mu \mathrm{g}$ ampicillin/ $\mathrm{ml}$. Plasmids from selected recombinants were sequenced with pscCMV forward (5'-TGGTGCAAATCAAAGAACTG-3') and reverse (5'-GTTTATTGVAGCTTATGG-3') primers and one (pGM-22) contained the correct nucleotide sequence.

The Viral Vector Core at TRINCH uses a good manufacturing practice production facility to generate $\mathrm{rAAV}$ vectors for researchers at TRINCH and external academic collaborators. We provided pGM-22 to the viral vector core and they generated and purified the self-complementary rAAV serotype 9 vector that expressed viperin from the CMV immediate/early promoter at a titer of greater than $5 \times 10^{11}$ DNase-resistant particles (DRP)/ $\mathrm{ml}$. The Viral Vector Core also provided the self-complementa- 
ry recombinant adeno-associated serotype 9 vector that expressed $g f p$ from the CMV immediate/early promoter for our studies.

Detection of GFP and Viperin in Chinchillas Transduced with Selected rAAV Vectors

As proof of concept that chinchillas could be transduced with self-complementary recombinant adeno-associated vectors, juvenile chinchillas were challenged intranasally with $1 \times 10^{11} \mathrm{DRP}$ rAAV serotypes 5, 6, or 9 that expressed GFP. Each of these constructs was provided by the Viral Vector Core at TRINCH. One week later, animals were imaged via micro-computed tomography (GE Healthcare, Pittsburgh, Pa., USA) as described [33], sacrificed, and GFP production in the nasal cavity was detected with a Leica M165 FC fluorescent dissecting microscope (Buffalo Grove, Ill., USA); images were captured with a Spot RT3 camera (Sterling Heights, Mich., USA). Selected tissues were then recovered from the nasal cavity and fluorescence was detected and quantified with a Xenogen IVIS Spectrum system (Caliper Life Sciences, Hopkinton, Mass., USA).

To determine whether we could overexpress viperin in the chinchilla airways during RSV A2 infection, we established 3 cohorts of 4 juvenile chinchillas. We challenged 1 cohort intranasally with $1 \times 10^{11}$ DRP rAAV serotype 9 that expressed GFP, another with $1 \times 10^{11}$ DRP rAAV serotype 9 that expressed viperin, and the third cohort received a mock treatment of viral diluent. Both rAAV vectors were diluted with saline to $200 \mu \mathrm{l}$ prior to challenge of the chinchillas. Seven days later, we inoculated all chinchillas with $1 \times 10^{8}$ pfu RSV A2 i.n. and NPL were performed 2, 4, and 7 days after viral challenge. NPL fluid was stored at $-80^{\circ} \mathrm{C}$. Animals were sacrificed and viperin was detected via immunohistochemistry as described [31] except that we utilized a monoclonal anti-mouse viperin antibody diluted 1:200 and a 1:200 dilution of a goat anti-mouse secondary antibody conjugated with AlexaFluor 594 (Invitrogen). DAPI (4',6'diamino-2-pheynylindole) was used to label DNA. Sections were viewed using a Zeiss Axiovert 200M microscope with ApoTome, and images were captured with an AxioCam MRm camera. As a comparison for relative viperin expression in the nasal cavity, archived sections of upper airway tissues from naive chinchillas were also processed in the same manner. The relative pixel signal intensity of viperin labeling was quantified with NIH ImageJ software (http://rsbweb.nih.gov/ij/) across the entire image. Sections from 3 animals per cohort were analyzed in duplicate and the mean pixel intensity was reported relative to naive animals.

\section{Ability of Viperin to Exhibit Anti-RSV Activity in the}

Mammalian Upper Respiratory Tract

To determine the ability of viperin to impact replication of RSV in the upper airways, rAAV serotype 9-treated animals were inoculated as described in the previous section and NPL fluids were collected. The titer of RSV A2 in NPL fluids was determined by inoculation in HeLa cells [34]. HeLa cells, grown to approximately $80 \%$ confluence in microtiter plates, were incubated with $20 \mu \mathrm{l}$ NPL fluids that had been diluted in $80 \mu \mathrm{l}$ cell culture medium. Cells were incubated for $24 \mathrm{~h}$ at $37^{\circ} \mathrm{C}$ with $5 \%$ $\mathrm{CO}_{2}$, growth medium was removed and cells were fixed for 20 min in 3\% paraformaldehyde. Cells were washed 3 times with $100 \mu \mathrm{l}$ PBS and permeabilized for $20 \mathrm{~min}$ with $100 \mu \mathrm{l}$ of $0.1 \%$
Triton X-100 (Fisher Scientific) diluted in $1 \times$ PBS. Cells were again washed 3 times with PBS and blocked with a milk blocking solution (KPL Inc., Gaithersburg, Md., USA) diluted 1:20 with sterile water. A 1:200 dilution of rabbit anti-RSV antibody (Thermo Scientific, Rockford, Ill., USA) was added to wells, and microtiter plates were incubated at $25^{\circ} \mathrm{C}$ for $1 \mathrm{~h}$. The solution was decanted and unbound antibody was removed by washing 3 times with $1 \times$ PBS for 5 min each. A 1:200 dilution of goat anti-rabbit antibody conjugated with AlexaFluor 594 was used as the secondary antibody. Cells were viewed using an inverted microscope and the number of RSV antigen-positive cells determined. The dilution factor and inoculum volume were used to calculate the RSV titer.

\section{Statistical Analyses}

Statistical analyses were performed by comparing data groups using Student's t test with significance accepted at $\mathrm{p} \leq 0.05$. Analyses were performed using GraphPad Prism.

\section{Results}

\section{Analysis of Global RSV-Induced Transcriptional Changes in the Upper Airways}

To determine how RSV infection of the upper respiratory tract affected expression of mucosal effectors of innate immunity, we performed a microarray analysis using RNA isolated from the chinchilla nasopharynx. Chinchillas ( $n=4 /$ cohort) were inoculated with RSV A2 or diluent, and sacrificed either 4 or 7 days later. As the chinchilla genome had not yet been sequenced at the time we originated these studies, we utilized the whole human Agilent array as it is known that many chinchilla cDNA sequences share significant similarity to their human orthologs $[29,33,35,36]$. We found that RSV inoculation of animals resulted in $\geq 1.5$-fold downregulation of 44 genes on day 4 , and 42 on day 7 (online suppl. tables S1 and S2). We also found that 24 transcripts were upregulated $\geq 1.5$-fold on day 4 and 28 transcripts on day 7 after inoculation (online suppl. tables S3 and S4). Included in this list were several genes such as STAT-1, SOCS-1 (suppressor of cytokine signaling 1), and IRF-7, whose encoded protein products are known to be involved in the host response to RSV infection of epithelial cells [18, 37]. In addition to these well-described targets, the gene rsad2 which encodes the antiviral protein viperin was induced 3 -fold 4 days and 4.2 -fold 7 days after inoculation (online suppl. tables S3 and S4). As viperin inhibits replication of several viruses $[22,24]$, our data demonstrated that viperin was upregulated in response to RSV, likely as a host mechanism to protect the airways against this virus. 


\section{Effect of RSV on Viperin Protein Production in the}

Airways

We next determined whether the increase in rsad2 (viperin) mRNA resulted in a concomitant increase in viperin protein at mucosal surfaces. RSV-infected and mockchallenged chinchillas ( $n=2 /$ cohort) were sacrificed 4 days after challenge, and we determined the relative amount of viperin protein in nasopharyngeal mucosa by immunodetection and densitometry. Nasopharyngeal homogenates from chinchillas infected with RSV for 4 days showed a greater abundance of native viperin compared to samples obtained from mock-infected chinchillas (fig. 1, top panel). We also detected GAPDH from these same tissue samples as a control for the loading of equivalent amounts of protein and as a standard for normalization of viperin protein expression (fig. 1, middle panel). Densitometric analysis showed that RSV infection of chinchillas resulted in 67 and $83 \%$ increases in viperin expression (fig. 1, bottom panel) compared to controls. Our results indicated that upper respiratory tract infection with RSV increased expression of viperin transcripts and viperin protein in the mucosa of the chinchilla upper airways.

\section{Determination of Relative Expression of Viperin}

Protein in Chinchilla Tissues

We have previously demonstrated that challenge of chinchillas with RSV results in the detection of viral antigen in tissues of the airways that include nasoturbinates, ethmoid turbinates and nasopharynx 7 days after viral challenge [31,32]. Micro-computed tomography images that depict the relative location of theses tissues within the chinchilla nasal cavity are shown in figure $2 \mathrm{a}$. As viperin is a cytosolic protein and is not secreted [19], we reasoned that viperin would have to be expressed not only in the nasopharynx but also in other epithelial airway tissues to provide an effective defense against RSV. Via immunoblot, we found that native viperin was produced in the mucosa of the nasal septum, nasoturbinate, ethmoid turbinate, nasopharynx, eustachian tube, and the lung of naive chinchillas (fig. 2b, top panel). Expression of viperin was greatest in the lung compared to the other upper airway tissues evaluated. We did not find viperin produced in non-respiratory tissues such as the stomach (data not shown) or bladder (fig. 2b, top panel). These data showed that viperin was expressed in tissues that are permissible to RSV infection $[31,32]$ in the upper and lower respiratory tracts.

We next evaluated whether challenge of chinchillas with RSV resulted in upregulation of viperin not only in the nasopharynx but also in additional upper airway tis-

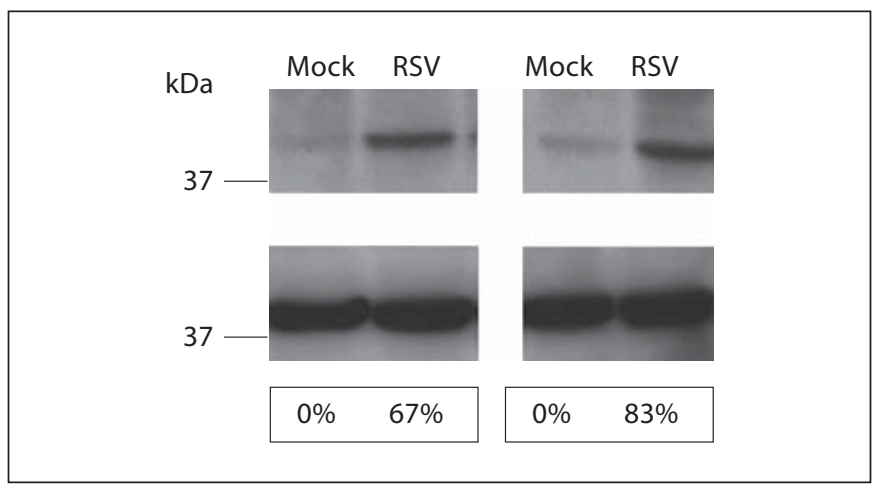

Fig. 1. Chinchillas ( $n=2 /$ cohort) were mock infected or challenged with $1 \times 10^{8}$ pfu RSV A2 and nasopharyngeal mucosa was collected 4 days later. Tissues were homogenized and viperin (top panel) or GAPDH (middle panel) was detected by immunoblot. Densitometric analysis of viperin protein abundance is shown and numbers represent the percent increase in viperin expression relative to mock-treated animals normalized to GAPDH expression (bottom panel). Challenge of chinchillas with RSV A2 resulted in increased expression of viperin protein compared to animals that were mock treated.

sues. Mucosae from a RSV-challenged chinchilla used in the microarray approach detailed in the section above were homogenized and proteins separated by SDS-PAGE. By densitometry, we determined that chinchillas challenged 4 days prior with RSV demonstrated at least an $84 \%$ increase in viperin expression in tissues that included the nasal septum, nasoturbinates, nasopharynx, and eustachian tube compared to naive animals (fig. $2 c$, top panel). Upregulation of viperin in animals challenged with RSV was least in the ethmoid turbinates (43\%) among the tissues that we evaluated. Abundance of GAPDH was used as a standard for normalization of viperin protein expression in these assays. These data indicated that upper airway infection with RSV increased expression of viperin in multiple tissues of the chinchilla upper airways.

To further define what cell types in the airways produce viperin, we utilized primary polarized chinchilla nasopharyngeal epithelial cells grown at the air-liquid interface for 40 days to induce cellular stratification and differentiation. Uninfected cells were fixed, embedded and immunolabeled for viperin. Whereas a control that did not include primary antibody showed only negligible labeling (data not shown), we readily detected viperin in cultured chinchilla nasopharyngeal epithelial cells (fig. $2 \mathrm{~d}$, top panel, green labeling). In parallel, we stained a serial section with hematoxylin and eosin and identified cell types based on their morphological differences. Ciliated respira- 
Fig. 2. a Micro-computed tomography was utilized to image the chinchilla nasal cavity in a sagittal (left panel) or coronal plane (right panel) with nasoturbinates, ethmoid turbinates, and the nasopharynx indicated by yellow arrows. b, c Tissues from the chinchilla upper and lower airways were homogenized, proteins were separated by SDS-PAGE and viperin (b, $\mathbf{c}$, top panel) was detected by immunoblot. Membranes were then stripped of antibodies and incubated with antisera directed against GAPDH as a control for loading of equal amounts of protein (b, c, bottom panel). d (top panel) Chinchilla nasopharyngeal epithelial cells were grown in vitro at an air-liquid interface to induce polarization and cellular stratification. Cultures were fixed, embedded in paraffin, sectioned, and incubated with a monoclonal antibody directed against viperin. Sections were then incubated with a secondary anti-mouse antibody conjugated to AlexaFluor 488 (green) and counterstained with DAPI to stain DNA (blue). d (bottom panel) HE-stained serial section of $\mathbf{d}$ (top panel) showing ciliated epithelial cells and goblet cells. Viperin was produced by ciliated epithelial cells in every airway tissue evaluated.

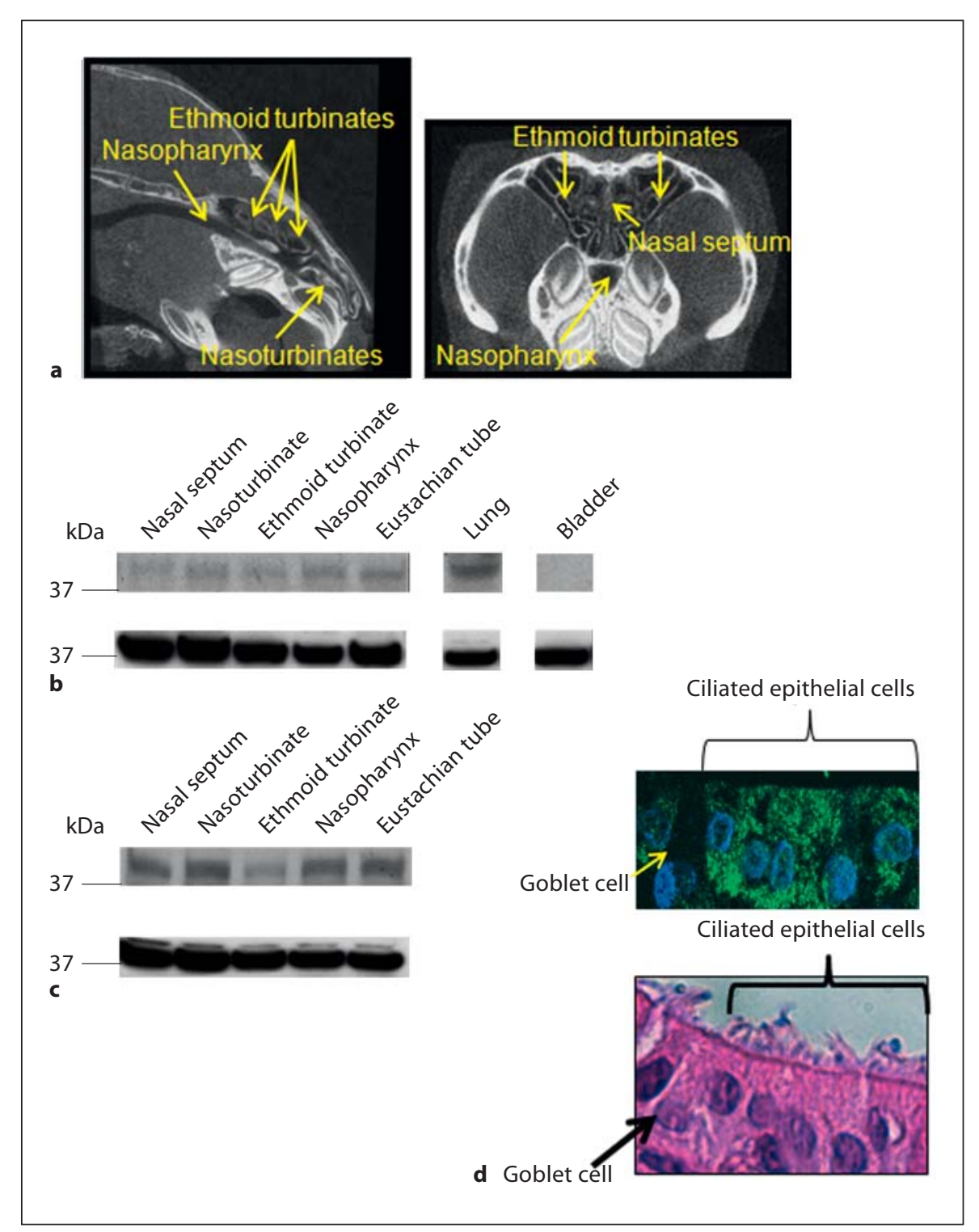

tory epithelial cells but not goblet cells stained with antibody to viperin, indicating that ciliated cells, not goblet cells, express detectable amounts of viperin (fig. $2 \mathrm{~d}$, bottom panel). Importantly, RSV exhibits tropism for ciliated human epithelial cells [12] and our data demonstrated that these cells derived from the upper airways expressed viperin endogenously.

\section{Effect of Viperin on RSV Replication in vitro}

HeLa cells are permissive for infection with RSV [38] and do not produce detectable amounts of viperin [19]. We therefore used these epithelial cells to determine if viperin expression affected RSV infection or replication.
We cloned the human viperin cDNA into an expression plasmid fused to an N-terminal V5 epitope tag and demonstrated our ability to express viperin in transiently transfected HeLa cells by PCR and immunoblot (online suppl. fig. S1A and S1B).

HeLa cells were therefore transfected with the viperin construct or a vector control, and inoculated with recombinant GFP-expressing RSV (rgRSV) at an MOI $=1$. The number of GFP-positive cells was determined $48 \mathrm{~h}$ later, and transfected cells that were mock treated served as a negative control. We demonstrated by flow cytometry that approximately $62 \%$ of cells treated with transfection reagent alone and then rgRSV were positive for GFP, where- 


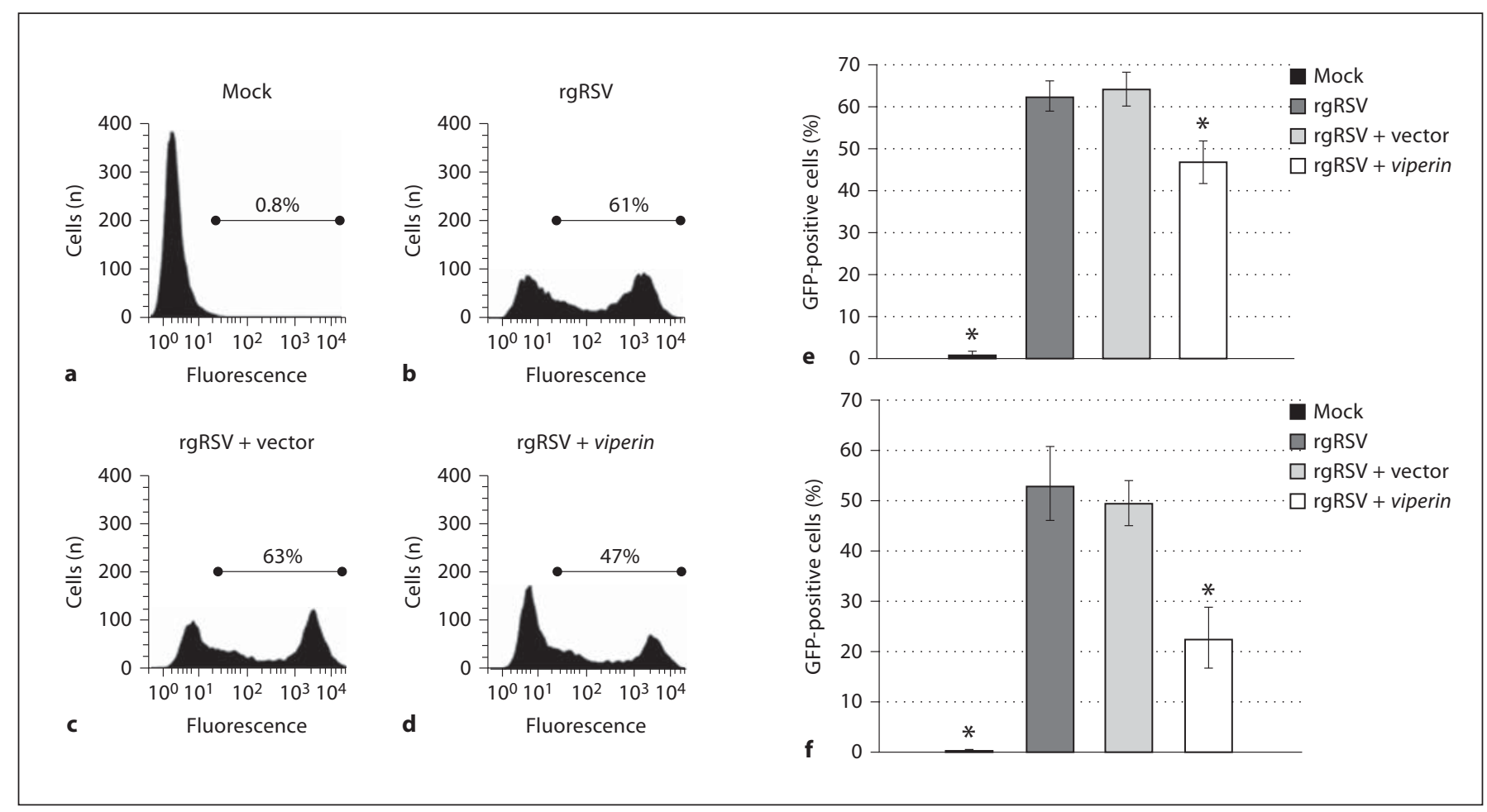

Fig. 3. HeLa cells or A549 cells were incubated with cell culture medium or transiently transfected with either vector alone or a viperin overexpression construct. One day later, cells were mock treated or incubated with $\mathrm{rgRSV}$ at an $\mathrm{MOI}=1$. Two days later, HeLa or A549 cells were trypsinized and the percentage of infected cells was determined via flow cytometry. Representative flow-cytometric plots of the mean percentage of GFP-positive
HeLa cells for each of the conditions tested are shown (a-d). e, f Percentages of GFP-positive cells $(\mathrm{n}=3$; e) and GFP-positive A549 cells $(\mathrm{n}=3$; f; means $\pm \mathrm{SD}) .{ }^{*} \mathrm{p} \leq 0.05$, GFP-positive cells vs. cells with rgRSV alone. Overexpression of viperin resulted in an approximate $20-30 \%$ decrease in the percentage of RSV-infected epithelial cells. as uninoculated cells had a negligible number of positive cells (fig. 3a, b, e). Similarly, cells that were transfected with vector alone and incubated with rgRSV had a mean of $64 \%$ infected cells (fig. 3c, e). In contrast, transfection of cells with the viperin expression plasmid resulted in $20 \%$ fewer rgRSV-infected cells compared to controls (fig. 3d, e).

To next determine whether overexpression of viperin impacted on replication of RSV in a respiratory epithelial cell line, we repeated the approach used with HeLa cells except that we now utilized A549 human alveolar epithelial cells which express low levels of endogenous viperin (data not shown). As with HeLa cells, we first demonstrated our ability to express viperin in transiently transfected A549 cells by immunoblot (online suppl. fig. S1C). We next demonstrated that transient transfection of A 549 cells with our viperin overexpression construct resulted in a statistically significant $30 \%$ decrease in GFP-positive cells compared to rgRSV alone (fig. 3f). As we evaluated 10,000 total events in our flow-cytometric approach, these data showed that approximately 3,000 fewer cells were infected with rgRSV when viperin expression was augmented compared to $\mathrm{rgRSV}$ alone. These results provide the first evidence that increased expression of viperin inhibits RSV replication within at least two distinct epithelial cell targets.

\section{Ability to Overexpress Viperin in the Chinchilla Upper Airways}

Our in vitro results suggested that viperin could inhibit replication of RSV in the mammalian upper airways. We therefore developed a molecular approach to overexpress viperin in the respiratory tract of the chinchilla host to determine if we could achieve a similar outcome in vivo. Different serotypes of rAAV have distinct abilities to transduce airway tissues of mice and rats $[39,40]$. We first tested the ability of rAAV serotypes 5,6 , or 9 that expressed $g f p$ under control of the CMV promoter to trans- 
Fig. 4. Chinchillas were challenged with $1 \times$ $10^{11}$ DNase I-resistant particles of self-complementary rAAV serotype 9 that contained the $g f p$ transgene under the control of the CMV immediate/early promoter. Seven days later, animals were sacrificed and GFP expression in several upper airway tissues was detected with a Xenogen IVIS Spectrum system (a) or a fluorescent dissecting microscope (b). Insets Mucosae from an animal that was not challenged with rAAV and therefore did not express GFP (a). GFP expression was detected in every tissue evaluated, which suggested that we could utilize rAAV to overexpress viperin throughout the chinchilla upper airways.
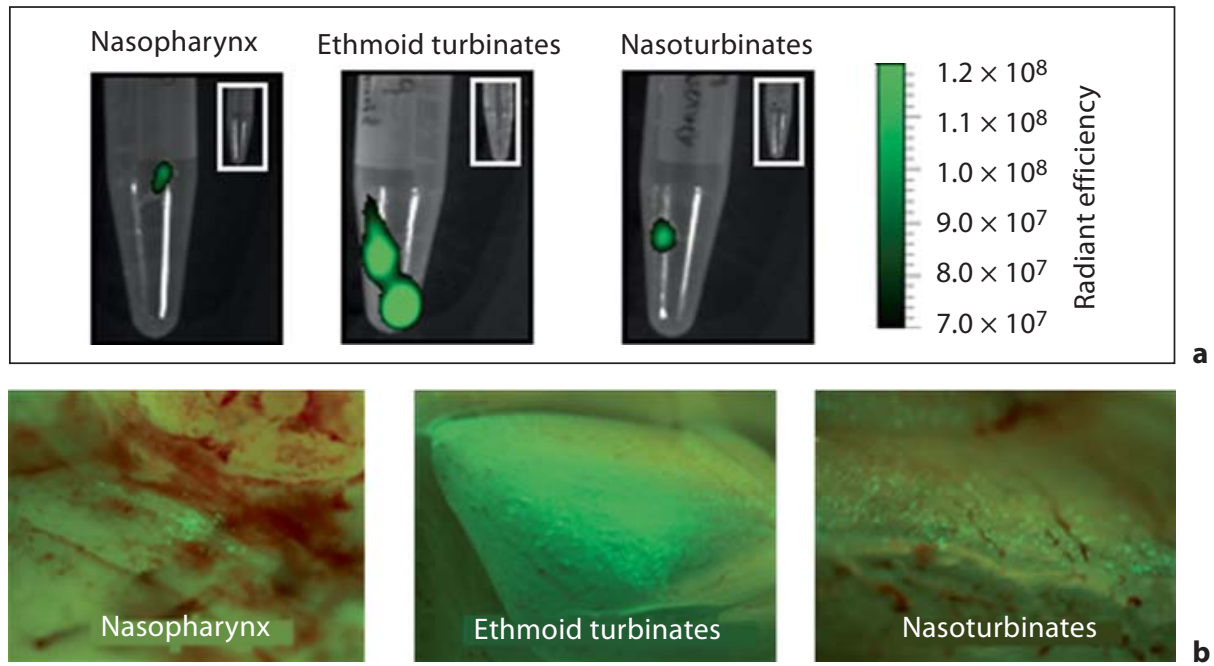

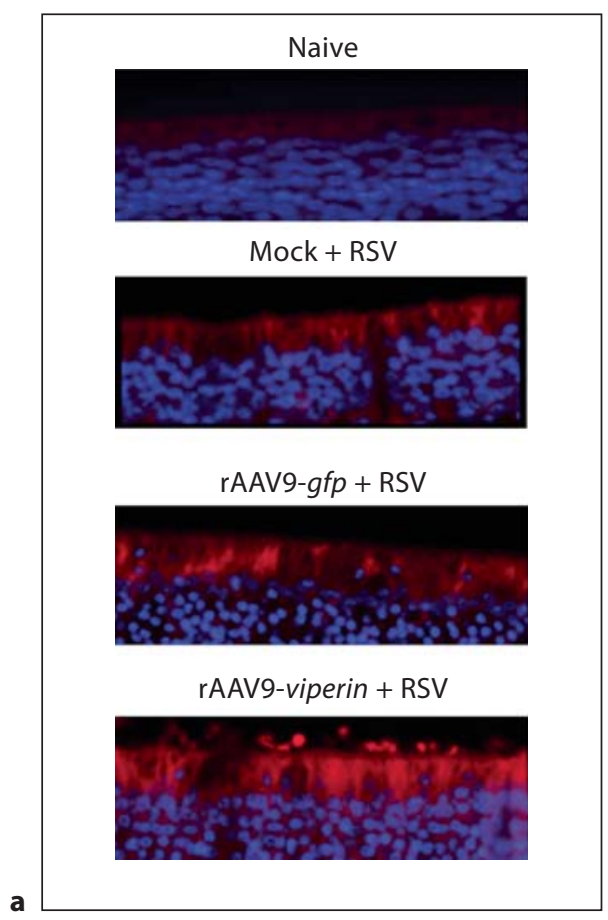

Fig. 5. Chinchillas ( $n=4 /$ cohort) were mock treated or inoculated with either rAAV that expressed GFP or viperin; 7 days later, animals were challenged with $1 \times 10^{8}$ pfu RSV A2. After 7 days, animals were sacrificed, the nasal cavity embedded and sections of tissue from the ethmoid turbinates were incubated with a monoclonal antibody directed against anti-mouse viperin (a) followed by a secondary anti-mouse antibody conjugated to AlexaFluor 594 (red) and counterstained with DAPI to stain DNA

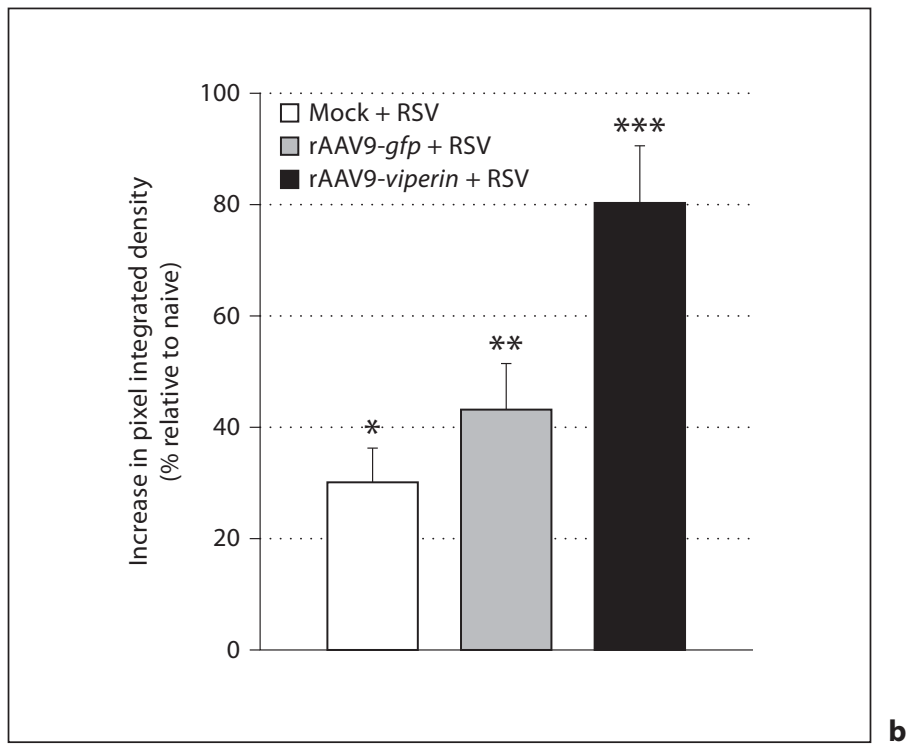

(blue). As a comparison, we also evaluated viperin expression in archived embedded sections of ethmoid turbinate tissue obtained from naive chinchillas. b ImageJ densitometry analysis of sections presented in a showing the relative expression of viperin among the cohorts with the naive animals set to a value of zero. Two images from 3 animals per cohort were analyzed. ${ }^{*} \mathrm{p} \leq 0.05$, ${ }^{* *} \mathrm{p} \leq 0.01,{ }^{* * *} \mathrm{p} \leq 0.0001$, vs. naïve animals. 
duce chinchilla upper airways. We detected modest fluorescence in nasoturbinates, ethmoid turbinates, or nasopharynx by fluorescence microscopy and Xenogen imaging 1 week after inoculation with either rAAV serotype 5 or 6 . In contrast, we observed robust GFP expression in all upper airway tissues evaluated from chinchillas that received rAAV serotype 9 (fig. 4). Xenogen imaging was used in this approach as this allowed us to quantitatively determine relative GFP expression among tissues as measured by radiant efficiency. The greatest signal that we observed was detected in the ethmoid turbinates followed by nasoturbinates and nasopharynx. As the same genome DNA was packaged in all three of these vectors and the same number of particles were inoculated for each vector, these results demonstrated that rAAV serotype 9 delivered to the nasal cavity of chinchillas resulted in the presence of $g f p$ in the greatest number of infected cells.

We next determined whether transduction of the chinchilla airways with rAAV serotype 9 that contained viperin would result in augmented production of viperin in the airways, in order to test the effect of viperin overexpression on RSV infection. Three cohorts of 4 animals each were established and administered vector diluent or $1 \times 10^{11}$ DRP of rAAV serotype 9 that expressed GFP or viperin. Seven days later, all chinchillas were challenged intranasally with RSV A2, and NPL fluids were collected 2,4 , and 7 days after inoculation with virus. The NPL samples were utilized to quantify RSV infectivity in the analysis presented in the section below. One week after challenge with RSV, animals were sacrificed, the nasal cavity was embedded and sections of ethmoid turbinate mucosae were prepared. As a comparison, we also evaluated viperin expression in archived embedded sections of the nasal cavity from naive chinchillas. Immunofluorescence microscopy revealed that in the absence of rAAV transduction, ethmoid turbinates were sites for viperin production (fig. 5a, top two panels) and that RSV increased viperin protein production thus confirming our earlier data obtained by immunoblot analysis (fig. 2b, c). Transduction of chinchillas with rAAV serotype 9-gfp followed by RSV A2 resulted in a modest (9\%) increase in viperin expression compared to RSV-challenged animals that were not transduced (fig. 5). In contrast, delivery of rAAV serotype 9-viperin to the chinchilla nasal cavity resulted in statistically significant ( $\mathrm{p} \leq 0.001) 51$ and $80 \%$ increases in viperin protein expression compared to RSVchallenged animals and naive animals, respectively. These data showed that targeted delivery of rAAV9-viperin augmented expression of viperin at a site of RSV replication in the upper airways (fig. 5).

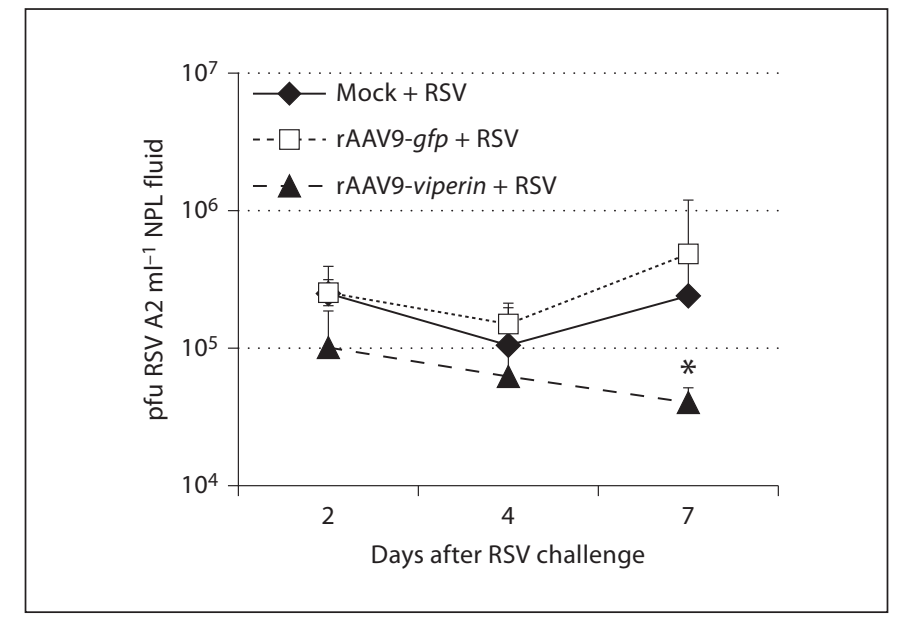

Fig. 6. Chinchillas were challenged as in figure 5 and NPL fluids were collected 2, 4, and 7 days after viral challenge. The concentration of RSV A2 in NPL fluids was determined by incubation of a serial dilution of sample with HeLa cells and staining for RSVpositive cells 1 day later. ${ }^{*} \mathrm{p} \leq 0.05$, RSV A2 titer vs. mock-treated animals. Overexpression of viperin resulted in decreased titers of RSV in chinchilla NPL fluids compared to controls. These data provided evidence that viperin exhibited anti-RSV activity in vivo.

\section{Effect of Viperin on RSV Replication in vivo}

To now determine if overexpression of viperin inhibited replication of RSV A2 in chinchilla upper airways, we compared viral titers in NPL fluids from animals among the 3 cohorts. Chinchillas that were mock treated or that received $\mathrm{rAAV}$ serotype 9-gfp before challenge with RSV A2 had titers of $2.5 \times 10^{5} \mathrm{pfu} / \mathrm{ml}$ after 2 days (fig. 6). Chinchillas that received rAAV9-viperin before RSV A2 showed a 58\% reduction in viral titer at this early time point, a result that approached statistical significance $(\mathrm{p}=0.1)$. A similar trend was observed 4 days after RSV A2 challenge, wherein animals that overexpressed viperin exhibited a diminished concentration of RSV A2 in NPL fluids compared to the other 2 cohorts. By day 7 , animals that were mock treated or that received rAAV serotype 9- $g f p$ before RSV A2 inoculation exhibited an increase in viral titer to approximately $5 \times 10^{5} \mathrm{pfu} / \mathrm{ml}$. In contrast, animals that received first $\mathrm{rAAV}$-viperin and then RSV A2 showed approximately 1 log less RSV A2 in NPL fluids, a statistically significant result (fig. 6; $\mathrm{p}=$ 0.05 ). These data indicated that overexpression of viperin in the upper airways results in a diminished ability of RSV to replicate in mucosal tissues of the mammalian airways. 


\section{Discussion}

RSV is a significant health problem for young children and the elderly, but RSV-induced diseases of the upper airways are typically not life threatening. Clinical data show frequent recurrence of RSV infection in young children, and the importance of RSV is underscored by recent studies that found RSV to be the predominant viral pathogen in terms of predisposition to bacterial otitis media [41]. In addition, recurrent upper airway infections enhance the chances of developing disease in the lower airways, which can be severe or even fatal in the pediatric population. We therefore initiated experiments to gain a greater understanding of RSV pathogenesis and the complex transcriptional host response to RSV in upper airways. Here, we have identified viperin as a gene that is upregulated in the upper airways of the chinchilla in response to RSV and have demonstrated both in vitro and in vivo that expression of this effector of innate immunity protects host cells against RSV infection.

We first utilized microarray analysis to determine the host response to RSV in nasopharyngeal mucosae of the chinchilla. In these experiments, we found that several genes implicated in the IFN response to virus, such as IRF-7 [42] and STAT-1 [18, 43], were upregulated in chinchilla upper airways. The number of transcripts up- or downregulated in response to RSV that we reported likely underestimates the complete transcriptional response of the host, as we used a human genechip for our analysis. The number of probes that showed a hybridization signal above background in the microarray was approximately $45 \%$ and therefore more than half of the oligonucleotides on the chip did not have sufficient similarity to bind their chinchilla counterpart sequences. Nonetheless, we detected a significant increase in the expression of the IFNinducible gene viperin in response to RSV at both 4 and 7 days after viral challenge. Although not highlighted in their studies, Janssen et al. [18] demonstrated that viperin (also called $r s a d 2$ ) was upregulated in the mouse lung 3.7fold 1 day after challenge, and Schuurhof et al. [37] demonstrated that viperin expression was increased in the lung 5.6-fold 5 days after viral challenge. These results are in agreement with our data that showed increased viperin expression in the chinchilla upper respiratory tract 4 and 7 days after RSV challenge and suggest that the RSV-dependent upregulation of viperin is conserved within the upper and lower airways and in different animal models of RSV disease. In addition, these data demonstrate that viperin is upregulated early after viral challenge and that augmented expression is maintained during RSV infec- tion, likely as an attempt by the host to limit viral replication.

We have previously demonstrated that RSV infection of chinchillas results not only in viral antigen in the nasopharynx but also in several upper airway tissues in a time-dependent manner [31]. Here we have found viperin was produced by ciliated epithelial cells and that this protein could be detected in all chinchilla tissues tested, including the nasoturbinates and eustachian tube. In addition, we demonstrated that chinchillas challenged 4 days prior with $1 \times 10^{8}$ pfu RSV A2 upregulated viperin protein production in every upper airway tissue evaluated. As RSV infects all of these tissues [31, 32], viperin could be mobilized to defend each of these sites from RSV.

To begin to address the ability of viperin to protect epithelial cells from RSV infection, we used an in vitro approach to transfect HeLa or A549 cells with a plasmid that contained the cDNA that encoded human viperin. We subsequently incubated cells with $g f p$-expressing RSV and determined the percentage of cells that could be infected. We demonstrated that the mean percentage of GFP-positive cells was nearly $20 \%$ less when HeLa cells were transfected with viperin and that this value increased to $30 \%$ when evaluated in A549 cells compared to controls. We speculate that the greater reduction in RSV-infected A549 cells, compared to HeLa cells, may be due in part to the endogenous expression of viperin in A549 cells, which is absent in HeLa cells [19]. As HeLa and A549 cells are typically not transfected at a high efficiency, the decreased number of infected cells that we observed in both cell types likely reflects inhibition of RSV in the transfected cells, which suggests that the ability of viperin to decrease RSV infectivity is quite efficient. Of note, generation of a cell line in which all cells were transfected with the overexpression construct would likely show an even greater impact on viperin-mediated anti-RSV activity. Although not addressed here, we speculate that the ability of viperin to inhibit replication of RSV in epithelial cells could involve disruption of viral assembly through altered lipid raft organization, similar to what is observed with the viperin inhibition of influenza A virus replication [22].

We expanded our investigation on the ability of viperin to exhibit anti-RSV activity to further include an in vivo model of upper airway infection. We found that rAAV serotype 9 was superior to serotypes 5 and 6 for transduction of the chinchilla airways. The rAAV serotype 5 and 6 receptor is sialic acid $[40,44,45]$ while the rAAV serotype 9 binds galactose moieties on the cell surface [39], which suggests that this sugar is more available for the viral par- 
ticles to bind in the chinchilla airways. Transduction of the human viperin gene by rAAV serotype 9 resulted in increased production of viperin compared to controls, as determined by immunodetection of this protein in upper airway tissues. Animals that were transduced with rAAV serotype 9-viperin and challenged with RSV 1 week later had reduced titers of RSV in NPL fluids at every time point evaluated $(2,4$, and 7 days), with the greatest impact 7 days after viral challenge. This is the first report of viperin protecting the airways against RSV replication.

Recent evidence has demonstrated that viperin is expressed not only in respiratory epithelium but also in other cell types such as dendritic cells, macrophages, and neutrophils [46]. As RSV infection mobilizes all of these cell types [47-49], viperin is likely not only an effector of innate immunity operational at mucosal surfaces but also contributes to the systemic response to RSV. This possibility is supported by the finding that viperin expression is significantly upregulated in the blood of human volunteers naturally infected with RSV [50].
Collectively, our evidence supports the conclusion that viperin plays an important role in the innate immune defense against RSV and likely limits RSV replication in many cellular targets. An increased understanding of the molecular mechanism of the anti-RSV activity mediated by viperin may enable the development of novel therapeutic strategies against this clinically important pathogen of the respiratory tract.

\section{Acknowledgments}

The authors would like to thank Dr. Peter White and the Biomedical Genomics Core at TRINCH for expert technical assistance with microarray analysis. We also thank Dr. K. Reed Clark and the Viral Vector Core at TRINCH for production of rAAV that expressed human viperin. Thanks go also to Dr. Peter Cresswell for his generous gift of antiserum to detect viperin and Jennifer Neelans for preparation of the manuscript. This work was supported by R01DC05847 and R01DC006468 to L.O.B.

\section{References}

1 Hall CB: Respiratory syncytial virus and parainfluenza virus. N Engl J Med 2001;344: 1917-1928.

$\checkmark 2$ Nair H, Nokes DJ, Gessner BD, Dherani M, Madhi SA, Singleton RJ, O’Brien KL, Roca A, Wright PF, Bruce N, Chandran A, Theodoratou E, Sutanto A, Sedyaningsih ER, Ngama M, Munywoki PK, Kartasasmita C, Simoes EA, Rudan I, Weber MW, Campbell H: Global burden of acute lower respiratory infections due to respiratory syncytial virus in young children: a systematic review and meta-analysis. Lancet 2010;375:1545-1555.

$>3$ Simoes EA: Respiratory syncytial virus infection. Lancet 1999;354:847-852.

$\checkmark 4$ Krusat T, Streckert HJ: Heparin-dependent attachment of respiratory syncytial virus (RSV) to host cells. Arch Virol 1997;142: 1247-1254.

$\checkmark 5$ Hallak LK, Spillmann D, Collins PL, Peeples ME: Glycosaminoglycan sulfation requirements for respiratory syncytial virus infection. J Virol 2000;74:10508-10513.

-6 Tayyari F, Marchant D, Moraes TJ, Duan W, Mastrangelo P, Hegele RG: Identification of nucleolin as a cellular receptor for human respiratory syncytial virus. Nat Med 2011;17: 1132-1135.

$>7$ Chaiwatpongsakorn S, Epand RF, Collins PL, Epand RM, Peeples ME: Soluble respiratory syncytial virus fusion protein in the fully cleaved, pretriggered state is triggered by exposure to low-molarity buffer. J Virol 2011; 85:3968-3977.
$>8$ Jeffree CE, Brown G, Aitken J, Su-Yin DY, Tan BH, Sugrue RJ: Ultrastructural analysis of the interaction between $\mathrm{F}$-actin and respiratory syncytial virus during virus assembly. Virology 2007;369:309-323.

$\checkmark 9$ Schlender J, Bossert B, Buchholz U, Conzelmann KK: Bovine respiratory syncytial virus nonstructural proteins NS1 and NS2 cooperatively antagonize alpha/beta interferon-induced antiviral response. J Virol 2000; 74:8234-8242.

10 Moore EC, Barber J, Tripp RA: Respiratory syncytial virus (RSV) attachment and nonstructural proteins modify the type I interferon response associated with suppressor of cytokine signaling (SOCS) proteins and IFN-stimulated gene-15 (ISG15). Virol J 2008;5:116.

-11 Spann KM, Tran KC, Chi B, Rabin RL, Collins PL: Suppression of the induction of alpha, beta, and lambda interferons by the NS1 and NS2 proteins of human respiratory syncytial virus in human epithelial cells and macrophages (corrected). J Virol 2004;78: 4363-4369.

12 Zhang L, Peeples ME, Boucher RC, Collins PL, Pickles RJ: Respiratory syncytial virus infection of human airway epithelial cells is polarized, specific to ciliated cells, and without obvious cytopathology. J Virol 2002;76: 5654-5666.
13 Groskreutz DJ, Monick MM, Powers LS, Yarovinsky TO, Look DC, Hunninghake GW: Respiratory syncytial virus induces TLR3 protein and protein kinase $\mathrm{R}$, leading to increased double-stranded RNA responsiveness in airway epithelial cells. J Immunol 2006;176:1733-1740.

14 Kurt-Jones EA, Popova L, Kwinn L, Haynes LM, Jones LP, Tripp RA, Walsh EE, Freeman MW, Golenbock DT, Anderson LJ, Finberg RW: Pattern recognition receptors TLR4 and CD14 mediate response to respiratory syncytial virus. Nat Immunol 2000;1:398-401

15 Monick MM, Yarovinsky TO, Powers LS, Butler NS, Carter AB, Gudmundsson G, Hunninghake GW: Respiratory syncytial virus up-regulates TLR4 and sensitizes airway epithelial cells to endotoxin. J Biol Chem 2003;278:53035-53044.

-16 Liu P, Jamaluddin M, Li K, Garofalo RP, Casola A, Brasier AR: Retinoic acid-inducible gene I mediates early antiviral response and Toll-like receptor 3 expression in respiratory syncytial virus-infected airway epithelial cells. J Virol 2007;81:1401-1411.

17 Katze MG, He Y, Gale M Jr: Viruses and interferon: a fight for supremacy. Nat Rev Immunol 2002;2:675-687.

18 Janssen R, Pennings J, Hodemaekers H, Buisman A, van Oosten $\mathrm{M}$, de Rond $\mathrm{L}, \mathrm{Oz}_{-}$ turk K, Dormans J, Kimman T, Hoebee B: Host transcription profiles upon primary respiratory syncytial virus infection. J Virol 2007;81:5958-5967. 
19 Chin KC, Cresswell P: Viperin (cig5), an IFN-inducible antiviral protein directly induced by human cytomegalovirus. Proc Natl Acad Sci USA 2001;98:15125-15130.

-20 Fitzgerald KA: The interferon inducible gene: viperin. J Interferon Cytokine Res 2011;31:131-135.

-21 Helbig KJ, Lau DT, Semendric L, Harley HA, Beard MR: Analysis of ISG expression in chronic hepatitis $\mathrm{C}$ identifies viperin as a potential antiviral effector. Hepatology 2005; 42:702-710

22 Wang X, Hinson ER, Cresswell P: The interferon-inducible protein viperin inhibits influenza virus release by perturbing lipid rafts. Cell Host Microbe 2007;2:96-105.

-23 Jiang D, Guo H, Xu C, Chang J, Gu B, Wang L, Block TM, Guo JT: Identification of three interferon-inducible cellular enzymes that inhibit the replication of hepatitis $\mathrm{C}$ virus. J Virol 2008;82:1665-1678.

24 Jiang D, Weidner JM, Qing M, Pan XB, Guo $\mathrm{H}$, Xu C, Zhang X, Birk A, Chang J, Shi PY, Block TM, Guo JT: Identification of five interferon-induced cellular proteins that inhibit West Nile virus and dengue virus infections. J Virol 2010;84:8332-8341.

-25 Zhang Y, Burke CW, Ryman KD, Klimstra WB: Identification and characterization of interferon-induced proteins that inhibit alphavirus replication. J Virol 2007;81:1124611255.

-26 Chan YL, Chang TH, Liao CL, Lin YL: The cellular antiviral protein viperin is attenuated by proteasome-mediated protein degradation in Japanese encephalitis virus-infected cells. J Virol 2008;82:10455-10464.

-27 Rivieccio MA, Suh HS, Zhao Y, Zhao ML, Chin KC, Lee SC, Brosnan CF: TLR3 ligation activates an antiviral response in human fetal astrocytes: a role for viperin/cig5. J Immunol 2006;177:4735-4741.

28 Proud D, Turner RB, Winther B, Wiehler S, Tiesman JP, Reichling TD, Juhlin KD, Fulmer AW, Ho BY, Walanski AA, Poore CL, Mizoguchi H, Jump L, Moore ML, Zukowski CK, Clymer JW: Gene expression profiles during in vivo human rhinovirus infection: insights into the host response. Am J Respir Crit Care Med 2008;178:962-968.

-29 McGillivary G, Mason KM, Jurcisek JA, Peeples ME, Bakaletz LO: Respiratory syncytial virus-induced dysregulation of expression of a mucosal beta-defensin augments colonization of the upper airway by non-typeable Haemophilus influenzae. Cell Microbiol 2009;11:1399-1408.

-30 McGillivary G, Ray WC, Bevins CL, Munson RS Jr, Bakaletz LO: A member of the cathelicidin family of antimicrobial peptides is produced in the upper airway of the chinchilla and its mRNA expression is altered by common viral and bacterial co-pathogens of otitis media. Mol Immunol 2007;44:24462458 .
31 Grieves JL, Jurcisek JA, Quist B, Durbin RK, Peeples ME, Durbin JE, Bakaletz LO: Mapping the anatomy of respiratory syncytial virus infection of the upper airways in chinchillas (Chinchilla lanigera). Comp Med 2010;60:225-232.

32 Gitiban N, Jurcisek JA, Harris RH, Mertz SE, Durbin RK, Bakaletz LO, Durbin JE: Chinchilla and murine models of upper respiratory tract infections with respiratory syncytial virus. J Virol 2005;79:6035-6042.

33 McGillivary G, Bakaletz LO: The multifunctional host defense peptide SPLUNC1 is critical for homeostasis of the mammalian upper airway. PLoS One 2010;5:e13224.

-34 Techaarpornkul S, Barretto N, Peeples ME: Functional analysis of recombinant respiratory syncytial virus deletion mutants lacking the small hydrophobic and/or attachment glycoprotein gene. J Virol 2001;75:68256834.

35 Kerschner JE, Erdos G, Hu FZ, Burrows A, Cioffi J, Khampang P, Dahlgren M, Hayes J, Keefe R, Janto B, Post JC, Ehrlich GD: Partial characterization of normal and Haemophilus influenzae-infected mucosal complementary DNA libraries in chinchilla middle ear mucosa. Ann Otol Rhinol Laryngol 2010; 119:270-278.

36 Kerschner JE, Horsey E, Ahmed A, Erbe C, Khampang P, Cioffi J, Hu FZ, Post JC, Ehrlich GD: Gene expression differences in infected and noninfected middle ear complementary DNA libraries. Arch Otolaryngol Head Neck Surg 2009; 135:33-39.

-37 Schuurhof A, Bont L, Pennings JL, Hodemaekers HM, Wester PW, Buisman A, de Rond LC, Widjojoatmodjo MN, Luytjes W, Kimpen JL, Janssen R: Gene expression differences in lungs of mice during secondary immune responses to respiratory syncytial virus infection. J Virol 2010;84:9584-9594.

38 Barretto N, Hallak LK, Peeples ME: Neuraminidase treatment of respiratory syncytial virus-infected cells or virions, but not target cells, enhances cell-cell fusion and infection. Virology 2003;313:33-43.

39 Bell CL, Vandenberghe LH, Bell P, Limberis MP, Gao GP, Van Vliet K, Agbandje-McKenna M, Wilson JM: The AAV9 receptor and its modification to improve in vivo lung gene transfer in mice. J Clin Invest 2011;121:24272435 .

40 Davidson BL, Stein CS, Heth JA, Martins I, Kotin RM, Derksen TA, Zabner J, Ghodsi A, Chiorini JA: Recombinant adeno-associated virus type 2, 4, and 5 vectors: transduction of variant cell types and regions in the mammalian central nervous system. Proc Natl Acad Sci USA 2000;97:3428-3432.
1 Patel JA, Nguyen DT, Revai K, Chonmaitree $\mathrm{T}$ : Role of respiratory syncytial virus in acute otitis media: implications for vaccine development. Vaccine 2007;25:1683-1689.

-42 Phipps S, Lam CE, Mahalingam S, Newhouse M, Ramirez R, Rosenberg HF, Foster PS, Matthaei KI: Eosinophils contribute to innate antiviral immunity and promote clearance of respiratory syncytial virus. Blood 2007; 110:1578-1586.

43 Pennings JL, Schuurhof A, Hodemaekers HM, Buisman A, de Rond LC, Widjojoatmodjo MN, Luytjes W, Kimpen JL, Bont L, Janssen R: Systemic signature of the lung response to respiratory syncytial virus infection. PLoS One 2011;6:e21461.

-44 Walters RW, Yi SM, Keshavjee S, Brown KE, Welsh MJ, Chiorini JA, Zabner J: Binding of adeno-associated virus type 5 to 2,3-linked sialic acid is required for gene transfer. J Biol Chem 2001;276:20610-20616.

$45 \mathrm{Wu}$ Z, Miller E, Agbandje-McKenna M, Samulski RJ: $\alpha 2,3$ and $\alpha 2,6 \mathrm{~N}$-linked sialic acids facilitate efficient binding and transduction by adeno-associated virus types 1 and 6. J Virol 2006;80:9093-9103.

46 Hinson ER, Joshi NS, Chen JH, Rahner C, Jung YW, Wang X, Kaech SM, Cresswell P: Viperin is highly induced in neutrophils and macrophages during acute and chronic lymphocytic choriomeningitis virus infection. J Immunol 2010;184:5723-5731.

-47 Everard ML, Swarbrick A, Wrightham M, McIntyre J, Dunkley C, James PD, Sewell HF, Milner AD: Analysis of cells obtained by bronchial lavage of infants with respiratory syncytial virus infection. Arch Dis Child 1994;71:428-432.

48 Gill MA, Palucka AK, Barton T, Ghaffar F, Jafri H, Banchereau J, Ramilo O: Mobilization of plasmacytoid and myeloid dendritic cells to mucosal sites in children with respiratory syncytial virus and other viral respiratory infections. J Infect Dis 2005;191:11051115.

49 Halfhide CP, Flanagan BF, Brearey SP, Hunt JA, Fonceca AM, McNamara PS, Howarth D, Edwards S, Smyth RL: Respiratory syncytial virus binds and undergoes transcription in neutrophils from the blood and airways of infants with severe bronchiolitis. J Infect Dis 2011;204:451-458.

50 Zaas AK, Chen M, Varkey J, Veldman T, Hero AO 3rd, Lucas J, Huang Y, Turner R, Gilbert A, Lambkin-Williams R, Oien NC, Nicholson B, Kingsmore S, Carin L, Woods CW, Ginsburg GS: Gene expression signatures diagnose influenza and other symptomatic respiratory viral infections in humans. Cell Host Microbe 2009;6:207-217. 